\title{
EFFICACY OF CORONALLY ADVANCED FLAP TECHNIQUE WITH COLLAGEN MATRIX MUCODERM IN COVERING MULTIPLE RECESSIONS - PRELIMINARY RESULTS
}

\author{
Rafał Wiench', Anita Ilków², Łukasz Gilowski ${ }^{3}$ Justyna Kulczycka', Patrycja Stelmańska-Bekus' ${ }^{1}$, Dariusz Skaba', \\ Tadeusz Faustyn Krzemiński ${ }^{4}$ \\ 'Department of Periodontal Disease and Oral Mucosa, School of Medicine with the Division of Dentistry in Zabrze, Medical University of Silesia \\ in Katowice, Poland \\ 2 Private practice, Opole, Poland \\ ${ }^{3}$ Private practice, Gliwice, Poland \\ ${ }^{4}$ Chair and Department of Pharmacology in Zabrze, School of Medicine with the Division of Dentistry in Zabrze, Medical University of Silesia \\ in Katowice, Poland
}

\begin{abstract}
InTRODUCTION: Gingival recession is defined as an apical shift of the gingival margin, causing exposure of the root surface of the tooth. Surgical techniques of treatment gingival recession are indicated for unesthetic, exposed, and/or painful root surfaces. The coronally advanced flap (CAF) together with a connective tissue graft is regarded as the gold standard but an alternative xenogenic material would reduce a discomfort of patients and provide for sufficient available tissue.

ОвJеCтIVEs: The aim of this case series study was to evaluate the efficacy of root coverage using the CAF technique with collagen matrix for the treatment of multiple recessions of Miller classes I and II over a short period of time.

MATERIAL AND METHODs: Twelve patients were enrolled in the study. Clinical recordings were obtained at baseline and after 3 months: RD (recession depth), RW (recession width), KTW (keratinized tissue width), CAL (clinical attachment level), CEJ-MGJ recorded from the CEJ (cement-enamel junction) to the MGJ (mucogingival junction), PD (depth of gingival sulcus) and RA (recession area). The percentage of average recession coverage (ARC) and the percentage of complete covered recessions (CRC) were evaluated 3 months after the surgery.

RESULTS: Significant decreases were recorded according to all of the parameters of recessions in comparison to the baseline measurements. The recession depth decreased from $2.0 \mathrm{~mm}$ to $0.5 \mathrm{~mm}$. The mean ARC was $87 \%$ and CRC $47 \%$.

ConcLusions: Xenogenic collagen matrix combined with the CAF technique led to a satisfactory ARC and an insignificant increase in the width of the keratinized tissue, but it yielded a lower, unsatisfactory CRC.
\end{abstract}

KEY WORDS: gingival recession, collagen matrix, mucogingival surgery.

J Stoma 2018; 71, 6: 478-482

DOI: https://doi.org/10.5114/jos.2018.85563

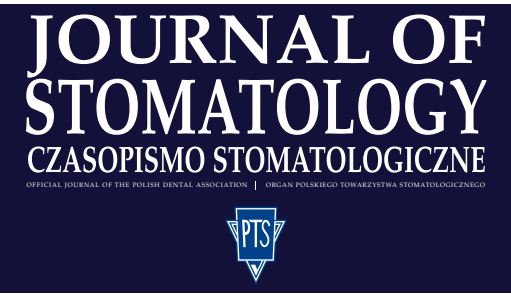

ADDRESS FOR CORRESPONDENCE: $d r$ n. med. Rafał Wiench, Department of Periodontal Disease and Oral Mucosa, School of Medicine with the Division of Dentistry in Zabrze, Medical University of Silesia in Katowice, Pl. Traugutta 2, 41-800 Zabrze, Poland, phone: +48 3227172 17, e-mail: wident@op.pl 


\section{INTRODUCTION}

The etiology of gingival recessions includes periodontal disease, thin biotype, malposition of the tooth in the dental arch, muscle attachment and mechanical trauma [1]. As a result, root surface exposure is frequently associated with esthetic complaints, root hypersensitivity and difficulties in achieving optimal plaque control [2].

The challenge for periodontal plastic surgical procedures is to achieve stable and complete root coverage with the attachment of the tissue margin at the cementoenamel junction and no bleeding on probing as well as a minimal probing depth of the healthy gingival sulcus $[1,3,4]$. However, the evidence on the minimal sufficient width and thickness of keratinized tissue needed to ensure long-term stability of marginal soft tissue has been controversial $[1,5]$.

Different surgical techniques (laterally positioned flap, or coronally advanced flap, tunnel technique) have been evaluated in the last few years to obtain root coverage with regard to predictability and the amount of root coverage achieved $[1,4]$.

The coronally advanced flap (CAF) together with a connective tissue graft (CTG) is regarded as the gold standard [4]. However, this technique has its limitations. The quantity of donor palatal tissue may be limited and moreover there is a degree of morbidity associated with CTG harvesting. For patients with multiple recessions, it can be difficult to harvest sufficient connective tissue from the palate, and multiple procedures may be necessary to achieve an optimal result [6]. Therefore, the availability of soft tissue substitutes appears useful for the treatment of these conditions. The question is whether the collagen matrix could be an alternative to a CTG for this technique. Recently a few xenogeneic materials have been tested for this indications (porcine collagen matrix, acellular dermal matrix) but the materials differ from each other in their properties [7-11]. Different efficacy for coverage of recessions and widening the keratinized tissue could be achieved with different products of porcine collagen matrix, for example with Mucograft and Mocoderm [12, 13]. The authors of the present study decided to use collagen matrix (Mucoderm) because this product was less studied as a substitute for CTG in coronally advanced flap technique. Mucoderm is an acellular sterilized collagen matrix derived from porcine dermis consisting of collagen types I and III and elastin [14]. Mucoderm was designed, developed and registered as a substitute for soft tissue graft for socket preservation, soft tissue augmentation and recession coverage procedures. So far, the present study is the first using Mucoderm in the CAF technique.

\section{OBJECTIVES}

The purpose of this clinical study was to evaluate the short-term (3 months) outcomes of root coverage using the coronally advanced flap technique with collagen matrix (Mucoderm) for the treatment of multiple recessions of Miller classes I and II.

\section{MATERIAL AND METHODS}

\section{STUDY DESIGN}

This case series study was conducted with the approval of the Local Ethical Committee (Institutional Review Board associated with the Medical University of Silesia, Katowice, Poland; protocol resolution no. KNW/0022/KBI/58/I/15). Patients eligible for the study were informed of the potential risks and benefits of the study and they signed informed consent statements. The treatment procedures were conducted from April 2016 to January 2018.

The primary outcome variable was the assessment of the CAL. The secondary outcome variables included the assessment of the recession depth and width, and keratinized tissue width.

\section{INTRA-EXAMINER REPRODUCIBILITY}

Ten patients not included in this study with diagnoses of multiple recessions were used to calibrate the examiner (AI). The examiner evaluated the patients on two occasions 24 hours apart. Clinical measurements (RD, RW, KTW, CAL, depth of gingival sulcus, distance from the cement-enamel junction to the mucogingival junction) were obtained with the same method as in the main study. The calibration was accepted if $95 \%$ of the recordings could be reproduced within a difference of $\leq 1 \mathrm{~mm}$.

\section{PATIENT POPULATION}

Twelve systemically healthy, non-smoking patients ( $\mathrm{F}-10, \mathrm{M}-2$; aged 23 to 50 years old) with 38 recessions (14 recessions in the mandible, 24 in the maxilla) of Miller classes I and II were selected from the patients in the Department of Periodontal and Oral Mucosa Diseases. All of the patients requested treatment for aesthetic reasons and/or dentin hypersensitivity of exposed roots.

The following inclusion criteria were applied:

- age of 18 to 60 years old;

- systemically healthy with no contraindications for periodontal surgery;

- absence of clinical signs of active periodontal disease;

- presence of a minimum of two adjacent periodontal recessions of Miller class I or II;

- presence of an identifiable CEJ (a step $\leq 1 \mathrm{~mm}$ at the CEJ level and/or presence of root abrasion, but with identifiable CEJ was accepted) [15]; and

- approximal plaque index (API) $\leq 15 \%$ and sulcus bleeding index $(\mathrm{SBI}) \leq 10 \%$. 

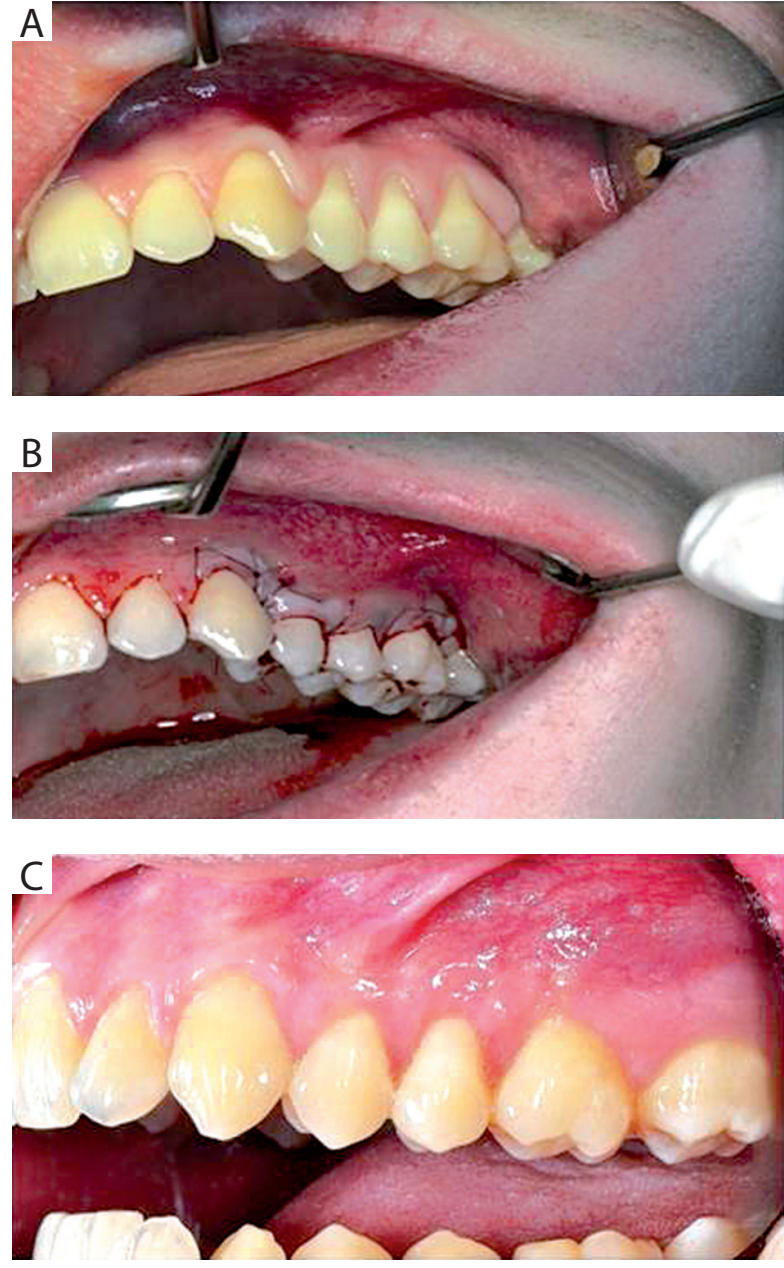

FIGURE 1. Photographic images before treatment (A), immediately after surgery (B) and 3 months after treatment (C)

Patients were excluded on the basis of the following criteria:

- pregnant or lactating;

- systemic disease with compromised healing potential or infectious disease;

- usage of medication known to affect gingival conditions (hyperplasia) or to interfere with healing (steroids);

- non-vital teeth, caries, prosthetic crowns or restorations involving the cemento-enamel junction area;

- tobacco smoking;

- drug or alcohol abuse.

Each patient received oral hygiene instructions and was motivated to maintain his or her oral health. The patients were instructed using a non-traumatizing buccal brushing technique (roll technique) with a soft toothbrush. All the participants were enrolled by one investigator (AI). The sulcus bleeding index and API were evaluated one month and one week before surgery in the patients enrolled in the study. The final examination confirmed the patient's compliance with the study enrollment criteria.

\section{CLINICAL MEASUREMENTS}

In all cases, preoperative clinical measurements were recorded with a CP15 periodontal probe, which had markings every millimeter. The measurements were rounded to the nearest $0.5 \mathrm{~mm}$. At baseline and at 3 post-surgically, the following measurements were recorded for each treated site: RD (recession depth) was measured from the mid-facial point of the CEJ to the free gingival margin; RW (recession width) was measured horizontally at the CEJ level; KTW (keratinized tissue width) was measured from the mucogingival junction to the gingival margin; CAL (clinical attachment level) was recorded from the CEJ to the deepest point of the gingival sulcus; CEJ-MGJ was recorded from the CEJ to the mucogingival junction; PD (depth of gingival sulcus) was recorded from the gingival margin to the deepest point of the gingival sulcus; and RA was the exposed root surface area (the area of a triangle with the recession width as the base and the recession depth as the height of the triangle). The periodontal recession coverage rating was calculated 3 months after surgery as the average percentage of root coverage (ARC) and the percentage of complete covered recessions (CRC). All pre- and postoperative measurements were obtained by the same previously calibrated examiner (AI).

\section{SURGICAL PROTOCOL}

The coronally advanced flap technique for multiple sites was used in the present study [15]. After local anesthesia the exposed root surfaces were planed using curettes to eliminate calculus and plaque present and rotary burrs to eliminate surface roughness. Then, using a no. 15C blade, a horizontal incision was made that extended to the papilla of both sides of the recessions. The horizontal incisions consisted of an oblique submarginal incision in the interdental areas continued with the intrasulcular incision at the recession defects. The oblique incisions started on both sides of the most affected tooth at the distance $\mathrm{RD}+1 \mathrm{~mm}$ from the tip of both papillae and ended at the gingival sulcus of the adjacent tooth at the level of the papilla's baseline. These incisions created new surgical papillae, which were raised with a split-thickness approach. Then the full-thickness flap was raised and again split with a deep and superficial incision to mobilize it and achieve a tension-free coronal position. The root surface with pre-surgical attachment loss was scaled with mini curettes. The anatomical papillae were deepithelialized. Previously rehydrated collagen matrix was placed in the recipient site to cover the recessions, positioned at the level of the cement-enamel junction (CEJ). Then, the matrix was stabilized separately to each tooth using 6-0 non-absorbable suspensory sutures. The mobilized mucous flap was then positioned at or coronally to the CEJ providing complete coverage 
of the collagen matrix. The flap was stabilized with 6-0 non-absorbable vertical mattress sutures (Figure 1).

\section{POST-SURGICAL INSTRUCTIONS}

Healing was inspected on the $1^{\text {st }}, 7^{\text {th }}$ and $14^{\text {th }}$ days after the surgical procedure by the surgeon. Sutures were removed after 2 weeks. All of the patients received instructions for proper home care. They did not brush the surgical site for 2 weeks but rinsed three times a day with a $0.12 \%$ solution of chlorhexidine gluconate. The patients were prescribed antibiotics because of the implanted xenogenic material. At all of the postoperative appointments, the areas were deplaqued and polished. Two weeks after surgery all of the patients were examined and instructed in tooth cleaning in the surgical area using a soft tooth brush and the roll technique. At 3 months after surgery, all of the clinical measurements were recorded again (AI).

\section{STATISTICAL ANALYSIS}

For all of the statistical evaluations the patient was maintained as the unit of measurement. Full mouth data analysis was performed for API and SBI, whereas defect site measurements were obtained for PD, CAL, RD, RW, KTW, CEJ-MGJ, and RA. Therefore, mean values and standard deviations (SD) for clinical variables were calculated for each patient per treatment. The Shapiro-Wilk test was used to determine the normal distribution of the studied parameters. Differences between measurements of clinical parameters before and after treatment were analyzed with the Wilcoxon test. A p-value less than 0.05 was considered statistically significant. All of the findings were analyzed using Statistica software, version 10.

\section{RESULTS}

Significant decreases were recorded according to all main parameters of recessions in comparison to the baseline measurements (Table 1 ). The recession depth decreased from $2.0 \mathrm{~mm}$ to $0.5 \mathrm{~mm}$. The recession width decreased from 3.5 to $1.4 \mathrm{~mm}$. An insignificant gain in the width of the keratinized tissue was also obtained from $3.2 \mathrm{~mm}$ to $3.4 \mathrm{~mm}$. The mean ARC was $87 \%$ after 3 months. The complete recession coverage (CRC) was $47 \%(18 / 38)$.

\section{DISCUSSION}

Recent studies have indicated that coronally advanced flap is one of the most effective surgical procedures and the addition of an autologous connective tissue graft increases the outcomes of these interventions [16]. A pilot study on single recession confirmed that combining CAF with collagen matrix led to good clinical results that compared favorably with those obtained using a connective tissue graft [7]. Recent studies on CAF technique in single recessions combined with CMX resulted in better outcomes than CAF alone without any addiction [10,17]. The root coverage after 6 months in above-mentioned studies was between $77 \%$ and $89 \%$, so similar to the present study, but the average gain in recession depth was larger (2.41 to $2.86 \mathrm{~mm})$ than in our study $(1.5 \mathrm{~mm})[7,10,17]$. Moreover, it is important that the results of these combined procedures are stable, which was confirmed with long-term observations $[17,18]$. A more recent study on single and multiple, adjacent recessions indicated that the addition of CMX to CAF resulted in a benefit in gingival thickness when compared with CAF alone [8, 19]. The data suggest that the clinical indications for the use of CMX are as follows: a) cases with contraindications to autologous CTG harvesting from the palate; b) cases where the patient and clinician are seeking to limit morbidity and are willing to accept a less than optimal outcome [19]. Moreover, the adjunctive application of CTG or CMX to recession coverage procedures may add a benefit especially at recession sites with thin marginal tissue [20]. These data support a conception of site-specific assessment in choosing surgical technique and potential addition of CTG or CMX [21]. The studies on multiple recessions using similar surgical technique and xenogenic collagen matrix or acellular dermal matrix resulted in comparable average gain in the level of gingival margin but the percentage of recession coverage was higher (93-94\% vs. $87 \%$ ) than in the present study and complete covered recessions also $[8,9]$.

A very important point of discussion is also the width of keratinized tissue. It is preferable to achieve a certain amount of attached gingiva for maintenance of the integrity of the dento-gingival junction [22-24]. A small, insignificant gain in the width of the keratinized gingiva was obtained in the present study. Most of the previous studies on CMX showed a larger increase of this parameter $[7,9,17]$. Also a previous study conducted at the author's department with the same material but used in

TABLE 1. Clinical parameters at baseline and after 3 months. Data are shown as means and standard deviation

\begin{tabular}{|l|c|c|c|}
\hline Parameter & \multicolumn{1}{c}{ Before } & After 3 months & $\boldsymbol{p}$-Wilcoxon test \\
\hline CAL & $2.8 \pm 0.8$ & $1.3 \pm 0.8$ & $<0.05$ \\
\hline RD & $2.0 \pm 0.8$ & $0.5 \pm 0.6$ & $<0.05$ \\
\hline RW & $3.5 \pm 0.9$ & $1.4 \pm 1.2$ & $<0.05$ \\
\hline RA & $3.9 \pm 2.1$ & $0.6 \pm 0.8$ & $<0.05$ \\
\hline KTW & $3.2 \pm 1.2$ & $3.4 \pm 0.9$ & $<0.05$ \\
\hline CEJ-MGJ & $5.2 \pm 1.1$ & $4.4 \pm 1.1$ & $<0.05$ \\
\hline
\end{tabular}

$C A L$ - clinical attachment level, $R D$ - recession depth, $R W$ - recession width, $R A$ - recession area, KTW - keratinized tissue width, CEJ-MGJ - cement-enamel junction - mucogingival junction 
combination with another surgical approach - the tunnel technique - resulted in a larger gain in the width of keratinized tissue [13].

\section{CONCLUSIONS}

To our knowledge, the study was the first to present the short-term effects of the coronally advanced flap technique with Mucoderm collagen matrix. Re-evaluation visits are planned and will indicate the stability of the results over the long term. The early results led the researchers to conclude that collagen matrix combined with CAF could lead to satisfactory average recession coverage of multiple Miller class I and II, but it yields lower, unsatisfactorily complete recession coverage.

\section{FUNDING SOURCE}

Medical University of Silesia. Contract number: KNW-1-042/N/5/0 (10.10.2015).

\section{CONFLICT OF INTEREST}

The authors declare no potential conflicts of interest with respect to the research, authorship, and/or publication of this article.

\section{References}

1. Miller PD. Regenerative and reconstructive periodontal plastic surgery: mucogingival surgery. Dent Clin North Am 1988; 32: 287-306.

2. Stefanini M, Marzadori M, Aroca S, et al. Decision making in rootcoverage procedures for the esthetic outcome. Periodontol 2000 2018; 77: 54-64.

3. Chambrone L, Pannuti CM, Tu YK, Chambrone LA. Evidencebased periodontal plastic surgery. II. An individual data metaanalysis for evaluating factors in achieving root coverage. J Periodontol 2012; 83: 477-490.

4. Chambrone L, Tatakis DN. Periodontal soft tissue root coverage procedures: a systemic review from the AAP Regeneration Workshop. J Periodotol 2015; 86 (2 Suppl): S8-S51.

5. Kennedy JE, Bird WC, Palcanis KG, Dorfman HS. A longitudinal evaluation of varying widths of attached gingiva. J Clin Periodontol 1985; 12: 667-675

6. Oates TW, Robinson M, Gunsolley JC. Surgical therapies for treatment of gingival recession. A systematic review. Ann Periodontol 2003; 8: 303-320.

7. McGuire MK, Scheyer ET. Xenogenic collagen matrix with coronally advanced flap compared to connective tissue with coronally advanced flap for the treatment of dehiscence - type recession defects. J Periodontol 2010; 81: 1108-1117.

8. Ahmedbeyli C, Ipci SD, Cakar G, et al. Clinical evaluation pf coronally advanced flap with or without acellular dermal matrix graft on complete defect coverage for the treatment of multiple gingival recessions with thin tissue biotype. J Clin Periodontol 2014; 41: 303-310.

9. Cardaropoli D, Tamagnone L, Roffredo A, Gaveglio L. Coronally advanced flap with and without a xenogenic collagen matrix in the treatment of multiple recessions: a randomized controlled clinical study. Int J Periodontics Restorative Dent 2014; 34: 97 102.
10. Moreira ARO, Santamaria MP, Silvério KG, et al. Coronally advanced flap with or without porcine collagen matrix for root coverage: a randomized clinical trial. Clin Oral Investig 2016; 20: 2539-2549.

11. Tonetti MS, Cortellini P, Pellegrini G, et al. Xenogenic collagen matrix or autologous connective tissue graft as adjunct to coronally advanced flaps for coverage of multiple adjacent gingival recession: Randomized trial assessing non-inferiority in root coverage and superiority in oral health-related quality of life. J Clin Periodontol 2018; 45: 78-88.

12. Aroca S, Molnar B, Windisch P, et al. Treatment of multiple adjacent Miller Class I and II gingival recessions with a Modified Coronally Advanced Tunnell (MCAT) technique and a collagen matrix or palatal connective tissue graft: a randomized, clinical trial. J Clin Periodontol 2013; 40: 713-720.

13. Cieślik-Wegemund M, Wierucka-Młynarczyk B, Tanasiewicz M, Gilowski Ł. Tunnel technique with collagen matrix compared with connective tissue graft for treatment of periodontal recession: a randomized clinical trial. J Periodontol 2016; 87: 1436-1443.

14. Pabst AM, Happe A, Callaway A, et al. In vitro and in vivo characterization of porcine acellular dermal matrix for gingival augmentation procedures. J Periodontal Res 2013; 49: 371-381.

15. Zucchelli G, Mounssif I, Mazzotti C, et al. Coronally advanced flap with and without connective tissue graft for the treatment of multiple gingival recessions: a comparative short- and longterm controlled randomized clinical trial. J Clin Periodontol 2014; 41: 396-403.

16. Cairo F, Nieri M, Pagliaro U. Efficacy of periodontal plastic surgery procedures in the treatment of localized facial gingival recessions. A systemic review. J Clin Periodontol 2014; 41 (Suppl 15): 544-562.

17. Jepsen K, Stefanini M, Sanz M, et al. Long-term stability of root coverage by coronally advanced flap procedures. J Periodontol 2017; 88: 626-633.

18. Mcguire MK, Scheyer ET. Long-term results comparing xenogeneic collagen matrix and autogenous connective tissue grafts with coronally advanced flaps for treatment of dehiscence - type recession defects. J Periodontol 2016; 87: 221-227.

19. Stefanini M, Jepsen K, De Sanctis M, et al. Patient reported outcomes and aesthetic evaluation of root coverage procedures: a 12 months follow-up of a randomized controlled clinical trial. J Clin Periodontol 2016; 43: 1132-1141.

20. Cairo F, Cortellini P, Pilloni A, et al. Clinical efficacy of coronally advanced flap with or without connective tissue graft for the treatment of multiple adjacent gingival recessions in the aesthetic area. A randomized controlled clinical trial. J Clin Periodontol 2016; 43: 849-856.

21. Cortellini P, Pini Prato G. Coronally advanced flap and combination therapy for Root coverage. Clinical strategies based on specific evidence and clinical experience. Periodontology 2000; 59: 158-184.

22. Kennedy JE, Bird WC, Palcanis KG, Dorfman HS. A longitudinal evaluation of varying widths of attached gingiva. J Clin Periodontol 1985; 12: 667-675.

23. Farnaoush A, Schonfeld SE. Rationale for mucogingival surgery: a critique and update. J West Soc Periodontol/Periodontal Abstracts 1983; 31: 125-130.

24. Schoo WH, Velden UVD. Marginal soft tissue recessions with and without attached gingiva. J Periodontal Res 1985; 20: 209-211. 